\title{
Designing for Mobility, Collaboration and Information use by Blue-Collar Workers
}

\author{
Jacqueline Brodie and Mark Perry \\ DISC, Brunel University
}

\section{INTRODUCTION}

The uptake of mobile phones in the UK has increased exponentially in the past two years, indicating that a wider range of users are now utilising mobile technologies in different contexts than ever before. Still little is known about how mobile technologies are used amongst different populations in specific contexts and this research addresses the context of work use by blue-collar workers with an aim to augmenting this with new mobile technologies better suited to their informational and communicative needs.

Most of the current public domain research into mobile device use practice concentrates primarily on professional workers (the ubiquitous 'mobile professional') and 'knowledge workers' (e.g. Bellotti and Bly, 1996; O'Hara et al. 2001). It seeks to discover how mobile technology, particularly Personal Digital Assistants (PDA's) and 'communicators', can be designed to help mobile professionals retain a sense of awareness of their workplace and work colleagues while they are away from their traditional workplaces. To a lesser extent, 'teens' (Ling, 2000) using SMS/ text messaging and novice users (e.g. Palen, 2000) are also examined, but there is very little understanding of the nature of other, and equally as important (in terms of the numbers of users and their importance to the economy), less well represented user groups.

\section{BACKGROUND TO THE AREA}

Specific research - and its concomitant development work - into the informational and communicative needs of mobile blue-collar workers (either skilled, semi-skilled or non-professional workers) is strange in its absence. Perhaps this research is not seen as 'sexy', or that these users are the commercially important 'early adopters' whatever the case, little has been done to redress the issue. This is a worrying trend in the design of technology - not only is it in a sense discriminatory (in that increasingly technological power is invested in the hands of managers, and not the workers), but also because it is ignoring a potentially large market. In the UK, it would be rare to find a self-employed blue-collar worker who did not use a mobile telephone for work. However, the kinds of other mobile technologies available to this cohort are extremely limited, as most of the current technologies are very much to do with building contact lists, diaries and other forms of documents used in knowledge work.
This lack of interest in blue-collar worker research is despite the lessons learned in Orr's study (1996) into mobile photocopy repair technicians, where he identified that the shared "war stories" of these technicians had a critical role to play in supporting their daily work practices. This lead to his recommendation that members of this 'community of practice' (Wenger, 1998) should be given radio equipment to enable them to remain in contact throughout the day to support each other in their repair tasks. Likewise, Luff and Heath's (1998) research looking at another mobile blue-collar community of practice, London underground station staff, identified the need for suitable mobile technology that could support their collaboration with other communities of practice such as the police when a suspect package is found in the station or with engineers and maintenance staff when they need to discuss possible solutions to technical problems that have occurred.

Work at the Viktoria Institute has been of interest in this area too, as they have looked at more non-traditional areas of mobile technology use (e.g. repairmen), with the intention of supporting this with mobile technology (Kristoffersen and Ljungberg, 1999). However, their research has focussed largely on the issues of the interface, rather than on the aspects of use in the work itself. Other technology developers, such as Symbol (www.symbol.com) have developed hardware solutions for the more rugged, industrial settings and in-vehicle logistical and navigational systems, whilst Psion (www.psion.com) have developed technologies for use in warehousing and transportation settings. However, these are largely process-based technologies and users have had little opportunity (or encouragement) to use them as cooperative or collaborative systems.

The blue-collar research we are carrying out at Brunel will benefit the developers of mobile computing and mobile telephony by providing them with an understanding of a significant, but largely invisible group of their users, their informational requirements, and potential avenues for the development of next generation devices to support them. This population of blue collar workers has so far also been opaque to network service providers, as their business uses are often hidden by service contracts that classify them as 'consumers' rather than 'business users' in customer databases. This is an increasing problem for phone networks, as the form of 'pay-as-you-go' telephone contracts do not even allow them to identify the name of the phone owner, let alone their profession. 


\section{FINDINGS FROM A PRELIMINARY STUDY}

We carried out a preliminary study into collaboration and information use in mobile work focussing on the communicative behaviour of five mobile business people. This research was carried out between November 2000 and February 2001 and involved a one and a half-hour contextual interview following a business trip taken by each of our participants. The key findings of our research were as follows:

Mobile collaboration ${ }^{3}$ is hard and more difficult to achieve than traditional remote collaboration in CSCW/groupware because of the organisational, social, cultural, cognitive and technical challenges of being mobile. We found business people tended to rely on the use of the mobile telephone to co-ordinate face-to-face meetings and document exchanges to complete their work activities when mobile. Achieving even this was difficult - as phone calls to other static or mobile colleagues often led to several rounds of fruitless voice mail messages before people were able to communication with each other synchronously.

There is the expectancy of immediacy - of 'anytime, anywhere' access to email, voice mail and other information sent by colleagues and clients despite the fact that mobile devices are seldom able to live up to this promise. This can cause anxiety and frustration on both the receiving and sending sides of electronic communications.

Despite an expressed desire by most of those interviewed to retain the boundaries between work and home-life there appeared a heavy spill over of work activities into homelife for mobile workers. This lead to discussions about what one of our participants termed "chalk-lining": where 'lines' are drawn around activities to distinguish them as work or home activities regardless of location or time of day (e.g. writing business emails on the trains at night or phoning friends in the car when travelling to and from business locations during the day).

After documenting in our preliminary research a whole host of problems that dog mobile white-collar 'communities of knowledge', we decided to turn our attention, in our full study, to look at mobile blue-collar 'communities of practice'. This is because if white-collar workers, with their wide array of technological resources to draw on find it hard to collaborate with their colleagues when mobile, what hope for successful collaboration

\footnotetext{
${ }^{3}$ We differentiate between two forms of action here; by collaboration we mean working on a shared task-based goal, whilst co-ordination involves a simpler form of information recall or articulation work (most usually agreeing where to meet, or breaking a task into parts).
}

could exist for blue-collar workers in their resource impoverished environments?

\section{MOBILE 'WORKSPACES' AND EMERGENT 'WORKPLACES' FOR BLUE-COLLAR WORKERS}

It has been pointed out that we cannot create a sense of 'space' through technology but only a sense of 'place'. Place is "socially constructed" through "social meaning, conventions and cultural understandings" etc. (Harrison and Dourish, 1996:69), and it seems clear that mobile technologies can be designed and used to provide a space for 'communities of practice. These virtual communities of practice would allow groups such as mobile blue-collar workers (of various kinds) to create their own sense of 'workplace' and to facilitate their opportunities to communicate, co-ordinate and ultimately collaborate no matter what they may be doing or wherever they may be.

Unfortunately, from the point of view of user-centred design, this neglect of research into how mobile bluecollar workers operate, the problems they face, and the strategies and techniques that they use to co-ordinate/ collaborate with mobile or static colleagues is a problem. Without this, how can designers and developers envision and create future mobile technology that will successfully support the dynamic, flexible and often urgent nature of the work that mobile blue-collar workers do? Fieldwork is desperately needed to ensure that the demanding nature of mobile blue-collar work is fully documented, and the physical constraints that any mobile blue-collar device will function under are examined. Attempts to design technologies that aim to support the communicative and collaborative activities of mobile blue-collar workers are likely to fail without this. Our research as outlined below seeks to address this deficit.

\section{TECHNOLOGY LANDSCAPE}

This work is happening against a changing technological landscape - the much hyped (and questionably valued) GPRS and 3G services are appearing closer to deployment, and Bluetooth and location-based technologies are nearing the marketplace. These will allow the always on, always visible, device connectivity technologies that may provide the basis for allowing the generation of a distributed and mobile community of practice. We are already examining how some of these could be applied in the design of technology with networked mobile handheld devices (iPaq), using high bandwidth, radio technology (WLAN). Over the next year we will be using these studies to design and evaluate prototype applications to support these user communities, and the results will feed into the next stage of mobile device design.

\section{RESEARCH AND DATA COLLECTION}

Following on from our recent research at Brunel University into the 'capture and communicate' activities of business people when they are mobile, our blue-collar 
research will draw on ethnographic and contextually grounded fieldwork techniques. It is intended to investigate the use of mobile voice and data connectivity in the real world settings of mobile blue-collar workers. This seeks to uncover not only the nature of blue-collar work, but also how mobile technology is integrated into the lives of blue-collar workers at present and what kinds of activities future technology could and should be supporting.

Our research entails the investigation of the mobile work activities of 15 blue-collar workers. Five blue-collar workers are being studied in-depth by detailing the activities they undertake in their everyday work as they are shadowed over a day using an ethnographically informed fieldwork approach (Agar, 1980). This is complemented through the use of unstructured contextual interviews (Beyer \& Holtzblatt 1997; Väänänen-VainioMattila and Ruuska 1998) for a further ten participants, who face questions regarding their use of artefacts, generated or collected in their work, to allow them to meet their informative, collaborative and communicative needs. Data collection and analysis will take place over the summer period and we will be in a position to discuss our findings at the workshop.

\section{ONGOING WORK (DATA COLLECTION AND ANALYSIS)}

As of $1^{\text {st }}$ August 2001, our research interviews are ongoing. We have conducted seven interviews so far with a range of blue collar workers: (1) a telecommunications engineer/technician, (2) one of several brothers in a family run firm of domestic gas installers, (3) a fax, printer, photocopy repair engineer, (4) an electric meter installer in homes and offices, (5) an electrician, (6) a mobile hairdresser, (7) a mobile cleaner, (8) a builder, (9) a painter and decorator, and (10) a plumber. All were highly mobile, working in a range of different sizes of organisations. We have so far only had time to conduct a preliminary analysis of some of these interviews, but the findings are revealing and early indications are that the participants' activities and needs appear very different to previous work on mobile professionals.

\section{PRELIMINARY FINDINGS}

For the most part, the people interviewed were heavy mobile phone users - (when this was not the case it was because their firms refused to pay their mobile phone bills). In fact, one of our participants recalled an occasion where she opted not to answer the phone during her working day and missed around 125 calls. Despite this use of telephone technology, many of our participants (although not all) were extremely resistant to using other forms of technology. Indeed, there was a great deal of diversity in the technological skills and task-based technical abilities of the participants. Whilst there were large differences between the interviewees, there were also a number of interesting similarities in aspects of the work they carried out. These similarities tended to revolve around their resolution of issues with regard to a) independence from and interdependence with others; $b$ ) their reliance on physical work (and their communication of information about what this is and its physical performance, which is typically not found in knowledge work) and, perhaps most noticeably, c) the culture of blue collar manual work.

\section{The mobile phone helps shape and support the way the job is done.}

An important point that arises from our data, analysed so far, is that our phone-using blue collar workers could not do the jobs they do - the way they do them now - without the aid of their mobiles. An illustration of this was related by the gas appliance installer, who pointed out that his family run firm would not have been able to grown the way it had without the mobile phone to handle the logistics of the day-to-day running of the business, as well as allowing the engineers to be contacted at a moment's notice by Housing Residency Associations for urgent jobs, such as when gas was smelt in a home. Our research participants used the phone to co-ordinate their activities with control rooms, their co-workers, their customers and their families and friends. However, accountability plays a part too in the mobile phone use of our participants demonstrated by our telecommunications engineers decision not to answer the phone one day during her working hours. Usually, she would only get two phone calls from her line manager during the day but on the day in question she received ten calls from him. Likewise, our photocopy repair engineer was required, by his superiors, to inform someone when he had finished a job, even when his callout centre was closed for the evening. This meant he often has to resort to texting one of his managers just to notify them of a completion of a call out.

In the small family business the gas engineers had a tendency to phone each other up directly with respect to small queries, bypassing the shop manager, because he was often busy selling domestic appliances to customers and unable to answer the phone the moment it rung. In larger businesses, however, our participants would be expected to go through the control room when trying to locate a colleague - although in practice, this was not always the case. Indeed, when the photocopy repairman had previously worked for Rank Xerox he had "instant messenger" on his laptop and he could freely talk (textbased) to his colleagues when on a job, which he found extremely useful for knowledge discovery. The telecommunications engineer would often use SMS text messaging to arrange lunch with colleagues or meet up to provide assistance on a job with less experienced colleagues.

\section{Capture and communication of information throughout the day}

In our small sample so far, our blue-collar workers need to capture and communicate a great deal of information 
during the day - although the bulk of information dealt with is "capture" type information. This information comes from central control rooms, their line managers, customers and other departments and communities that they work with. Much of it is numerical in nature e.g. job numbers; serial numbers of equipment being used; model numbers and phone numbers. At other times they need to access quite detailed and technical information which ranged from manuals to blue prints, plans and estimates for jobs; as well as looking at log books and seeing what kinds of repair work, and by whom, had previously been carried out on a machine. Some of the information received through the phone is also urgent in nature. Our mobile hairdresser recounted spending time on the phone with a hairdressing product help-line while they detailed how to correct a hair colouring session that had gone disastrously wrong.

The kinds of information that is carried around and needed by the participants in our research is illustrated in the photograph of the photocopier repairman's car shown below:

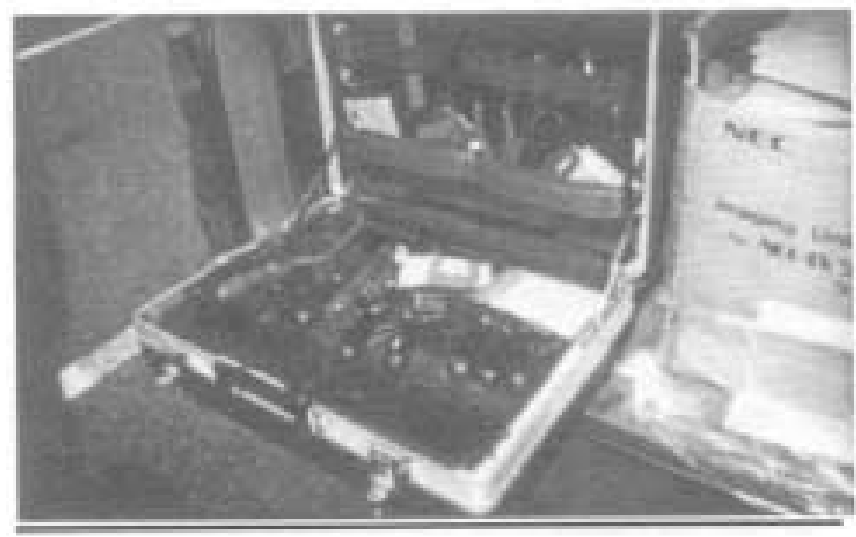

The photocopier repairman has to carry his toolbox everywhere with him. Shown also in the picture, next to his briefcase, is his box of manuals, which he needs when tackling a machine he is not familiar with.

Our participants often discussed during the interviews some easy way to record the information they captured during the day e.g. our telecommunications engineer thought that a recording memo facility on her phone would be useful so that once given a job number she could speak it into her phone to store this quickly (and without having to interact and access a more complex device manually). The photocopy repairman and I discussed the possibility, in the future, of a camera in a phone so the person he was taking advice from could see what he had done so far and also watch as he tried their suggestions to fix the device. The information given to our participants often had to be transformed by them before doing their job. For instance, our electrician recalled having to negotiate with a plumber about where to put his electric cables in respect to the plumber's pipes. Both saw the plans given to them as flexible artefacts - something that they could interpret and negotiate with others over - and through discussion they reached a mutually acceptable compromise to the problem, which had not been resolved in the initial blue prints. However, in one instance, when a large change to the initial building plans was felt necessary by our electrician he had to take such a request to the site manager to take back to the construction engineers for their final say so on the matter.

Another interesting finding, so far from our research, is that awareness of what others in their team are doing would be beneficial to our blue-collar workers. There appears no point, when dealing with something urgent, to phone someone in their team whose phone is already engaged or switched off. Some kind of "buddy" awareness scheme operated through the phone would be useful for our workers. Likewise, mobile phones that combine both two-way radios and mobile phone facilities may be a good option for those on building sites.

\section{The social role of the phone is evident among our blue collar users}

One of the participants related that his son recently bought a house and would not have been able to deal with the transaction and still be at his work, as an apprentice gas installer, had it not been for his mobile phone, which allowed him to communicate with his solicitors when he needed to. The electrician in our study also recounted a similar story when he purchased his flat, but in his case, although his girlfriend was able to deal with the solicitors calls through her work phone, she regularly called him with progress updates as they occurred. The telecommunications engineer and her step-daughters used SMS texting throughout the day - helping them to retain their familial bond - and the photocopy engineer phoned his wife several times a day to let her know his whereabouts and when he would be able to pick her up from work at the end of the working day. The mobile hairdresser also used the phone to ask her relatives to come and pick up her children, who often accompanied her to a customer's home, if they became tired or bored during her visit. Nearly all our participants discussed the benefits to be had of different ring tones for different callers. Most suggested that at the very minimum they would have two different ring tones on their phone if they could - one for home and one for work - thereby allowing them to bypass checking caller display before deciding to answer a call.

A second interesting social aspect of telephone use, was the use of the telephone by colleagues (as a user proxy), and particularly by apprentices: we heard about occasions when a more experienced apprentice would take phone messages for the participants (and fill in simple forms also) whilst their trainer was busy. Apprentices often phoned the customer help-lines (of appliances that were being fixed) to get information if there were problems, while the participants continued to work - this was because the customer help-lines could take up to an hour of waiting on hold and being passed from one person to 
another. The participants also reported apprentices answering the telephone for them whilst driving between sites and asking their head offices for directions or instructions.

\section{Flexibility}

Organisations often do not appreciate the communication flexibility that their mobile blue collar workers' need. Both the engineer and the electrician's company appreciated that they needed to use the phone for their work. Each company has tackled the problem differently. In the case of the engineer his company fitted him with an in-van phone, whilst in the case of the engineer his company had given him a BT Chargecard (although he owned a personal mobile phone). Both of these artefacts limited who the workers could contact in a day - limiting their contacts to other co-workers, bosses, their base and their storeroom/depot for supplies, etc. However, as the study has shown, there is a need for more flexible communication channels to be available for these mobile workers. For example, our engineer discussed contacting a work colleague who was having a day off to find out where a location of a job was. If it had not been for having a mobile phone, the engineer would have had to locate a telephone box to make his call instead of being able to make his call in the comfort of his van with his maps and notebooks easily at hand. Likewise, it was easier for him to get help about a meter repair when he was standing in front of the machine, instead of sitting in his car trying to remember the last state that he left the meter in. In a similar way, our electrician would have had to have found a public phone box to phone his boss about a problem or to phone up to order supplies when on site. Of course, the fact that the participant's supervisors in both organisations know the mobile phone numbers of both men and used it to contact them regularly reveals that individual managers, if not organisations, appreciate the value of being in contact all the time with their employees during the working day.

\section{RESEARCH QUESTIONS ARISING}

Below is a list of issues that we will continue to seek answers to in our ongoing research:

- Does co-ordination/ collaboration exist among bluecollar workers when mobile at present and if not why not? Is it the nature of the work that prohibits collaboration, the nature of the technology or a relationship between the two?

- Our recent research into mobile professionals identified a slackening off of the traditionally boundaries between home life and work life of our mobile white collar workers. Is this merging of home life and work life also present in the lives of mobile blue-collar workers? For example, when the laptop is switched "on" mobile workers often consider themselves to be working, no matter their location. Do blue-collar workers feel the same if their work mobile phones are on, or do they only feel they are working when the other traditional tools of their profession are in their hands and their work clothes are on?

- Is there a price to pay for awareness? How 'open' are our blue-collar workers to allowing others an awareness of their daily activities? What kind of activities would they allow/want others to be made aware of and who would those others be?

- How well does existing technology and the nontechnological artefacts used by mobile blue collar workers allow them to co-ordinate/collaborate their activities with each other and those others outside their communities of practice such as suppliers of materials, their families and friends.

- What are the special requirements that being both mobile and working in a hands-on job, such as building, mean for the design of technological devices in the future if they are to provide a suitable workspace to facilitate the creation or maintenance of a sense of shared workplace?

\section{Acknowledgements}

We would like to thank Niki Green and Richard Harper for their thoughts and contribution to the research - some of the background material stems from collaborations with them. We would also like to thank Symbian for their support in helping us carry out the work. This work was also financially supported in part by the by the EPSRC through Grant no. GR/RO9503/01 (Designing for Mobile and Distributed Work)

\section{REFERENCES:}

Agar, M.H. (1980) The professional stranger: an informal introduction to ethnography. London: Academic Press.

Bellotti, V. \& Bly, S. (1996) Walking away from the desktop computer: distributed collaboration and mobility in a product design team. In CSCW 96: Proceedings of the Conference on Computer-Supported Cooperative Work, September 17-20, Boston, Mass.: ACM Press. p. 209-218.

Beyer, H. \& Holtzblatt K (1997) Contextual Design, San Francisco: Morgan Kaufman.

Harrison, S. \& Dourish, P. (1996) Re-Place-ing Space: The role of Place and Space in Collaborative Systems. In CSCW 96: Proceedings of the Conference on ComputerSupported Cooperative Work, September 17-20, Boston, Mass.: ACM Press. p 67-76.

Kristoffersen, S. \& Ljungberg, F. (1999) Making Place" to Make IT Work: Empirical Explorations of $\mathrm{HCI}$ for Mobile CSCW. In proceedings of Group'99. ACM Press.

Ling, R. Norwegian teens, mobile telephony and SMS use in school. Available at $<$ http://www.telenor.no/fou/prosjekter/Fremtidens_Bruker 
e/seminarer/mobilpresentasjoner/Norwegian $\% 20$ teens $\% 20$ MTs\%20SMS\%20and\%20school\%20use\%20notat\%2072000.doc>

Luff, P. \& Heath, C. (1998). Mobility in Collaboration. In proceedings of Conference on Computer Supported Cooperative Work, November 14-18, Seattle, Washington, USA, p. 305-314. New York: ACM Press.

O'Hara, .K., Perry, M., Sellen, A.J. and Brown, B.A.T. (2001) Exploring the relationship between mobile phone and document use during business travel. In Brown, B., Green, N., Harper, R. (Eds) Wireless World: social and interactional implications of wireless technology. Springer Verlag.

Orr, J. E. (1996) Talking about Machines: An ethnography of a modern job. Cornell University Press.
Palen, L., Salzman, M \& Youngs Ed. (2000) Going wireless: behavior \& practice of new mobile phone users. In Proceeding of the ACM 2000 Conference on Computer Supported Cooperative Work, Philadelphia, PA, p 201 210. New York: ACM Press.

Vanaanen-Vainio-Mattila, K. \& Ruuska, S. (1998). User needs for mobile communication devices. First Workshop on HCI for Mobile Devices, Glasgow, Scotland, May 1998.

Wenger, E. (1998) Communities of Practice: Learning, Meaning and Identity. Cambridge University Press, Cambridge. 